\title{
THE INNER DEBRIS STRUCTURE IN THE FOMALHAUT PLANETARY SYSTEM*
}

\author{
Kate Y. L. Su ${ }^{1,6}$, George H. Rieke ${ }^{1}$, Denis Defrére ${ }^{1}$, Kuo-Song Wang ${ }^{2}$, Shih-Ping Lai ${ }^{3,6}$, David J. Wilner ${ }^{4}$, \\ RiK VAN Lieshout ${ }^{5}$, and Chin-Fei LeE ${ }^{2}$ \\ ${ }^{1}$ Steward Observatory, University of Arizona, Tucson, AZ 85721, USA; ksu@as.arizona.edu \\ 2 Institute of Astronomy and Astrophysics, Academia Sinica, P.O. Box 23-141, Taipei 106, Taiwan \\ 3 Institute of Astronomy, National Tsing Hua University (NTHU), Hsinchu 30013, Taiwan \\ ${ }^{4}$ Harvard-Smithsonian Center for Astrophysics, 60 Garden Street, Cambridge, MA 02138, USA \\ ${ }^{5}$ Anton Pannekoek Institute for Astronomy, University of Amsterdam, Science Park 904, 1098 XH Amsterdam, The Netherlands \\ Received 2015 September 23; accepted 2015 December 10; published 2016 February 8
}

\begin{abstract}
Fomalhaut plays an important role in the study of debris disks and small bodies in other planetary systems. The proximity and luminosity of the star make key features of its debris, like the water ice line, accessible. Here we present ALMA cycle $1,870 \mu \mathrm{m}(345 \mathrm{GHz})$ observations targeted at the inner part of the Fomalhaut system with a synthesized beam of 0 ". $45 \times 0$ "! 37 ( $\sim 3$ AU linear resolution at the distance of Fomalhaut) and an rms of $26 \mu \mathrm{Jy}$ beam ${ }^{-1}$. The high angular resolution and sensitivity of the ALMA data enable us to place strong constraints on the nature of the warm excess revealed by Spitzer and Herschel observations. We detect a point source at the star position with a total flux consistent with thermal emission from the stellar photosphere. No structures that are brighter than $3 \sigma$ are detected in the central $15 \mathrm{AU} \times 15 \mathrm{AU}$ region. Modeling the spectral energy distribution using parameters expected for a dust-producing planetesimal belt indicates a radial location in the range of $\sim 8-15 \mathrm{AU}$. This is consistent with the location where ice sublimates in Fomalhaut, i.e., an asteroid-belt analog. The $3 \sigma$ upper limit for such a belt is $<1.3 \mathrm{mJy}$ at $870 \mu \mathrm{m}$. We also interpret the 2 and $8-13 \mu \mathrm{m}$ interferometric measurements to reveal the structure in the inner $10 \mathrm{AU}$ region as dust naturally connected to this proposed asteroid belt by Poynting-Robertson drag, dust sublimation, and magnetically trapped nanograins.
\end{abstract}

Key words: circumstellar matter - planetary systems - radio lines: stars - stars: individual (Fomalhaut)

\section{INTRODUCTION}

The Fomalhaut debris disk has become the prototype for understanding the complexity of disk structures and their relationship to planetary systems. Owing to its proximity (7.7 pc) and luminosity $\left(17.4 L_{\odot}\right)$, many key features of its debris are easily accessible through optical, infrared, and millimeter ground- and space-based facilities. The prominent planetesimal ring located at $140 \mathrm{AU}$, analogous to the Kuiper Belt in our solar system, is particularly well studied (Holland et al. 1998; Kalas et al. 2005; Acke et al. 2012; Ricci et al. 2012). The sharp boundaries of this cold ( $50 \mathrm{~K})$ ring suggest gravitational shaping by unseen planets (Quillen 2006; Chiang et al. 2009; Boley et al. 2012). Intense collisions among the planetesimals in this ring grind large (parent) bodies into fine dust, forming a disk halo composed of $\sim$ micron-sized grains outside the cold ring (Acke et al. 2012; Kalas et al. 2013). The Fomalhaut disk has a warm $(\sim 150 \mathrm{~K})$, unresolved component near the star, first indicated by Spitzer $24 \mu \mathrm{m}$ imaging (Stapelfeldt et al. 2004) and later confirmed by Herschel imaging (Acke et al. 2012). This warm excess has been suggested as an ice-line planetesimal belt, analogous to the asteroid belt in our solar system (Su et al. 2013). Furthermore, the Fomalhaut system is thought to have a very hot $(\sim 1500 \mathrm{~K})$ exozodiacal dust population at $\sim 0.1 \mathrm{AU}$, revealed by groundbased interferometry at $2 \mu \mathrm{m}$ ( $K$ band; Absil et al. 2009). Interferometric data at $\sim 10 \mu \mathrm{m}$ detect a hot component near 1-2 AU (Lebreton et al. 2013; Mennesson et al. 2013). Therefore, the Fomalhaut system is the only system known to

\footnotetext{
* Fomalhaut is a triple system; here we refer to the Fomalhaut planetary system as the one around the primary star Fomalhaut A.

6 Visiting Scholar, Institute of Astronomy and Astrophysics, Academia Sinica, P.O. Box 23-141, Taipei 106, Taiwan.
}

possess all five debris zones (very hot, hot, warm, cold, and halo dust) defined by Su \& Rieke (2014).

In addition to the debris system around Fomalhaut, a combination of imaging where the warm component can be spatially separated from the cold component and mid-infrared spectroscopy has revealed similar inner warm components in other nearby systems, for example, $\epsilon$ Eri (Backman et al. 2009; Greaves et al. 2014), HR 8799 (Su et al. 2009; Matthews et al. 2014), and Vega (Su et al. 2013). Approximately $20 \%$ of unresolved debris disks show such warm components in their disk spectral energy distributions (SEDs; Morales et al. 2011; Ballering et al. 2013; Chen et al. 2014; Kennedy \& Wyatt 2014). Leftover parent bodies near the star generally have higher collisional velocities, i.e., shorter dynamical timescales, compared to those of more distant ones, as suggested in the fading of the warm components after a few hundred Myr (e.g., Gáspár et al. 2013). These components may be analogous to our asteroid belt but with far greater mass; however, compared to the cold Kuiper Belt analogs, they are much harder to characterize in exoplanetary systems. Identification and characterization of such warm components require very accurate extrapolation of the stellar spectrum and precise subtraction.

Not surprisingly, although it can be considered the prototype, the nature of the Fomalhaut warm component is controversial. Acke et al. (2012) propose that it originates from free-free emission of a postulated stellar wind that also creates the hot excess revealed in near-infrared interferometry. However, Su et al. (2013) draw from all the resolved images and the spectroscopy to argue that it is a planetesimal ring placed near the ice line and presumably created by processes occurring in other debris systems as well. In that case the Fomalhaut debris disk resembles the debris structures in our 
Table 1

ALMA Cycle 1 Observations of Fomalhaut

\begin{tabular}{|c|c|c|c|c|c|c|}
\hline $\begin{array}{l}\text { EB } \\
\text { Code }\end{array}$ & UT Date & Passband & $\begin{array}{c}\text { Calibrators } \\
\text { Flux }\end{array}$ & Phase & $\begin{array}{c}\text { Number of } \\
\text { Antennae }\end{array}$ & $\begin{array}{l}\text { PWV } \\
(\mathrm{mm})\end{array}$ \\
\hline $\mathrm{X} 4 \mathrm{a} 7$ & 2014 Apr 29 & $\mathrm{~J} 2258-2758$ & $\mathrm{~J} 2258-279$ & $\mathrm{~J} 2250-2806$ & 35 & 1.43 \\
\hline X11ad & 2014 May 21 & $\mathrm{~J} 2258-2758$ & J2258-279 & $\mathrm{J} 2250-2806$ & 32 & 1.10 \\
\hline $\mathrm{X} 135 \mathrm{f}$ & 2014 May 21 & $\mathrm{~J} 2258-2758$ & $\mathrm{~J} 2258-279$ & $\mathrm{~J} 2250-2806$ & 32 & 1.24 \\
\hline $\mathrm{Xe} 46$ & 2014 May 26 & $\mathrm{~J} 2056-4714$ & $\mathrm{~J} 2258-279$ & $\mathrm{~J} 2258-2758$ & 25 & 0.67 \\
\hline $\mathrm{X} 119 \mathrm{f}$ & 2014 May 26 & $\mathrm{~J} 2258-2758$ & J2258-279 & $\mathrm{J} 2250-2806$ & 25 & 0.72 \\
\hline
\end{tabular}

solar system-a dense Kuiper Belt whose inner edge is maintained by massive planets (Liou \& Zook 1999) and a more tenuous asteroid belt containing dust structure (zodiacal cloud) determined by both the giant and terrestrial planets (Dermott et al. 1994).

There is a tentative detection of an excess in the ALMA cycle 0 image of Fomalhaut (Boley et al. 2012), but less than the prediction by the free-free model, and the star was at the edge of the primary beam, so the excess flux is subject to the large uncertainty of the primary beam correction. Here we present a high-angular-resolution and high-sensitivity ALMA cycle 1 image centered at the star position and provide a strong constraint on the amount of excess emission in the inner part of the Fomalhaut system.

Section 2 of this paper describes the details of the ALMA observation and data reduction. In Section 3, we discuss the $870 \mu \mathrm{m}$ measurement near the star, evaluate the expected photospheric value of Fomalhaut at this wavelength, and rule out any compact free-free emission that originated from the star as the possible source of excess emission detected by Spitzer and Herschel. In Section 4.1 we review the properties of the inner $20 \mathrm{AU}$ region in Fomalhaut by assessing all available excess measurements and discussing their uncertainties. We conclude that a warm excess at the ice sublimation temperature $(\sim 150 \mathrm{~K}$, i.e., an asteroid-belt analog) is robustly detected, independent of the uncertainty of photospheric subtraction and the presence of a tentative, hot $(\sim 500 \mathrm{~K})$ excess. In Section 4.2, we present SED models for the ice-line asteroid belt around Fomalhaut and set constraints on its total flux at $870 \mu \mathrm{m}$, and we also show that a 13 AU narrow belt might be detected at $\lesssim 2 \sigma$ levels in the cycle 1 ALMA data. In Section 4.3, we propose a new interpretation for the hot excess detected by the Keck Interferometric Nuller (KIN) via linking the proposed asteroid belt to the dust structure in the inner $10 \mathrm{AU}$ region. Additional validation in terms of the derived dust mass and the sensitivity of the adapted grain parameters to our model are discussed in Section 5, followed by our conclusions in Section 6.

\section{OBSERVATIONS AND DATA REDUCTION}

Fomalhaut was observed with the ALMA $12 \mathrm{~m}$ array in Cycle 1 (program 2012.1.00238.S; PI: Lai) in five execution blocks at band 7 . Table 1 summarizes the detailed information for each execution, including the bandpass, amplitude, phase calibrators, and the estimated precipitable water vapor (PWV). The data taken on the second execution of 2014 May 21 did not pass the quality assurance procedure and thus are not included in the imaging and analysis study. A total on-source time of 172 minutes was achieved. The phase center is located at the star position (R.A. = 22:57:39.409, Decl. $=-29: 37: 22.423$ ) corrected for proper motion at the epoch of the observation.
Four $2 \mathrm{GHz}$ wide spectral windows centered at 337.987 , $339.924,350.002$, and $352.002 \mathrm{GHz}$ were used to measure the continuum emission with dual linear polarizations from the target source. The primary beam size (the half-power width of the Gaussian beam) is $18^{\prime \prime}$ at $345 \mathrm{GHz}$. The overall $u v$-coverage ranges from $23.0 \mathrm{k} \lambda$ to $737.7 \mathrm{k} \lambda$. Thus, our data are not sensitive to smooth emission structures larger than $\sim 9^{\prime \prime}$. The width of the outer belt is estimated to be $\sim 13-19$ AU (Boley et al. 2012), which is mostly resolved out by our high resolution ( $\sim 3 \mathrm{AU}$ in linear scale); therefore, our data only show a very faint trace of the outer belt along the minor-axis direction. The quasar J2258-279 was used as the amplitude calibrator in all execution blocks. The flux uncertainty is estimated to be $10 \%$.

All the data reduction and imaging processes were carried out with CASA 4.2.1 (McMullin et al. 2007). The final continuum image was obtained by performing multi-frequency synthesis (MFS) imaging. Clean boxes of the expected cold belt location (generated with reference to the Hubble Space Telescope $[H S T]$ image) and the central $5^{\prime \prime}$ for the warm belt were used in the image deconvolution process. A final image with an effective bandwidth of $8 \mathrm{GHz}$ centered at $345 \mathrm{GHz}$ was generated. The synthesized beam is $0.45 \times$ 0 ". 37 with position angle (P.A.) of $82^{\circ} .3$. The rms noise in the final MFS image is $26 \mu \mathrm{Jy}_{\text {beam }}{ }^{-1}$.

\section{ALMA OBSERVATIONAL RESULTS}

\section{1. $870 \mu \mathrm{m}$ Measurement}

Figure 1 (a) shows the central $6^{\prime \prime} \times 6^{\prime \prime}$ region of the map. A bright source, detected at the star position after taking the star's proper motion into account, has an FWHM of 0 ." $46 \times 0$ ". 38 at P.A. of $86^{\circ} .4$, consistent with being the star (also see Section 3.2). To uncover any faint structure that might be present around the star, we fitted a single point-source model to the data, subtracted it from the original data in the $u v$ plane, and then generated the residual map. We used uvmodelfit in CASA to determine the best-fit parameters for the point source: a total flux of $1.789 \pm 0.037 \mathrm{mJy}$. The quoted uncertainty is based on the fit, i.e., this number does not include other errors from flux calibration. The residual map is shown in Figure 1(b). No structures that are brighter than $3 \sigma$ are detected in the $2^{\prime \prime} \times 2^{\prime \prime}$ $(15 \mathrm{AU} \times 15 \mathrm{AU})$ region around the star after subtracting the best-fit point-source model. Assuming that the inner belt has a similar orientation to the outer belt, one would expect the disk ansae to be along the P.A. of $156^{\circ}$. There are two $\sim 2 \sigma$ blobs along that angle: one is $\sim 0$ !" 4 (3 AU) northwest of the star, and the other is $\sim 1$ !' 8 (14 AU) southeast of the star. Based on our SED model (see Section 4.2), the likely location of the inner belt is at radii of $\sim 8-15 \mathrm{AU}$, suggesting that the southeast blob could be part of the inner belt. Assuming that the inner belt is also inclined by $66^{\circ}$ like the outer one, but centered at the star 

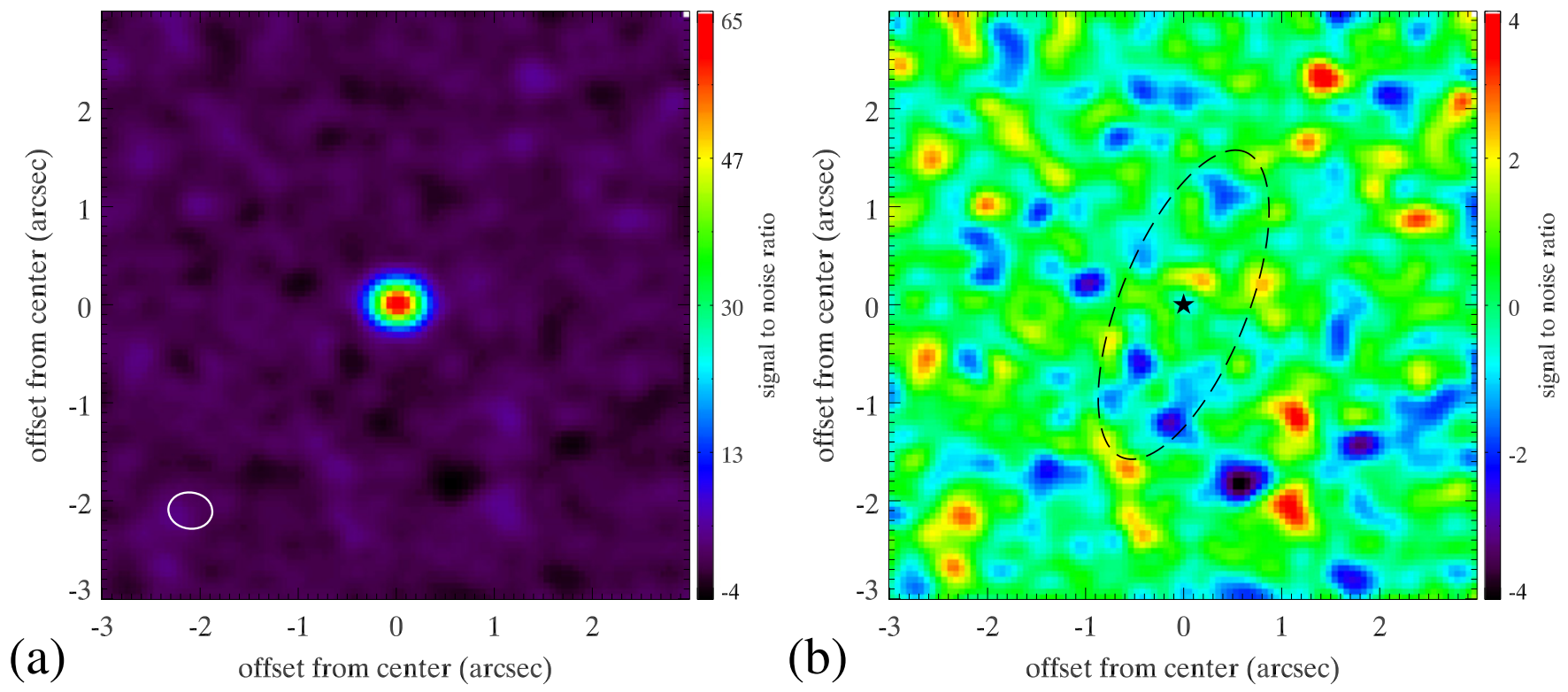

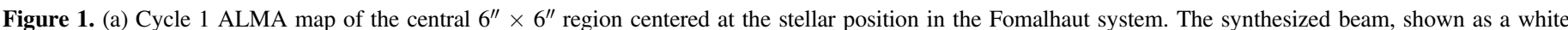

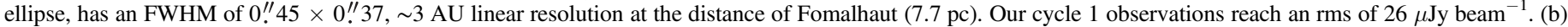

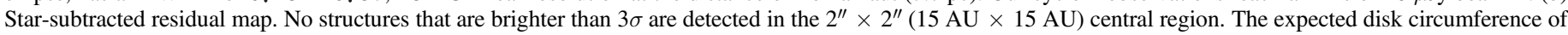

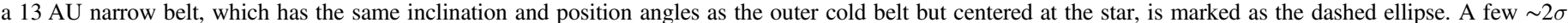
blobs are found along the ellipse.

(no offset), the ellipse on Figure 1(b) marks the boundary of a putative $13 \mathrm{AU}$ inner belt. Interestingly, there are a few $1 \sigma-2 \sigma$ blobs along the expected disk circumference, suggesting that this $13 \mathrm{AU}$ inner belt might be detected at $1 \sigma-2 \sigma$ levels (more discussion is given in Section 4.2). Although the $1 \sigma-2 \sigma$ positive blobs along the non-offset, expected $13 \mathrm{AU}$ disk circumference are intriguing, we note that there are also many $-2 \sigma$ blobs in the data as well. In summary, we do not find any significant extended structure around the star, and the reality of the putative $13 \mathrm{AU}$ belt needs future confirmation.

\subsection{Expected Photosphere Brightness at $870 \mu \mathrm{m}$ and a Constraint on the Unresolved Excess}

$\mathrm{Su}$ et al. (2013) evaluated all available optical to nearinfrared photometry and determined the stellar photospheric output to be $2.96 \mathrm{Jy}$ in the MIPS $24 \mu \mathrm{m}$ band. A simple Rayleigh-Jeans extrapolation then yields an estimate of $2.2 \mathrm{mJy}$ at $870 \mu \mathrm{m}$, significantly brighter (by $\sim 23 \%$ ) than the point source measured at the star position. The discrepancy likely comes from two sources: (1) the uncertainty of the ALMA absolute calibration, and (2) the uncertainty of Rayleigh-Jeans extrapolation.

The accuracy in absolute calibration for ALMA Cycle 2 is set at $10 \%$ for Band 7 (J. Mangum 2015, private communication). To verify the calibration, we used the solar analog method (Johnson 1965; Rieke et al. 2008) by comparing measurements of $\alpha$ Cen A with those of the Sun. We did this in two parts. First, we verified that $\alpha \mathrm{Cen} \mathrm{A}$ is a solar analog by comparing its colors with those of the Sun. Then, we computed the submillimeter flux of $\alpha$ Cen A using the solar relation between measured $24 \mu \mathrm{m}$ and submillimeter. We derived colors of $V-K_{s}=1.531 \pm 0.023, \quad V-[24]=1.584 \pm$ 0.027 , and $K_{s}-[24]=0.053 \pm 0.032$ using the available $K$ band measurement from Engels et al. (1981), $V$ band from Alekseeva et al. (1996), Mermilliod (1991), and Hipparcos, and $24 \mu \mathrm{m}$ measurement of $30.84 \mathrm{Jy}$ from Wiegert et al. (2014) after proper photometric system transformation and calibration to the zero points defined by Rieke et al. (2008). These colors are virtually identical to those of the Sun and establish $\alpha$ Cen A as a valid solar-analog star for the purpose of calibration.

We then used measurements of the solar brightness temperature to connect the mid-infrared measurements to the ALMA calibration at $870 \mu \mathrm{m}$. We adopt the brightness temperature at $24 \mu \mathrm{m}$ to be $4625 \mathrm{~K}$ (Model $\mathrm{M}$ in Vernazza et al. 1976) and the brightness temperature at $870 \mu \mathrm{m}$ to be $5470 \mathrm{~K}$ (Loukitcheva \& Nagnibeda 2000). Taking $30.84 \pm 0.76 \mathrm{Jy}$ as the $24 \mu \mathrm{m}$ flux of $\alpha$ Cen A (Wiegert et al. 2014), the scaling from the solar brightness temperatures suggests that $\alpha$ Cen A has a flux density of $28.9 \mathrm{mJy}$ at $872 \mu \mathrm{m}$. The error associated with this number is not straightforward to determine, but can be estimated as the quadratically combined error of $7 \%( \pm 300 \mathrm{~K}[6 \%]$ in the solar $870 \mu \mathrm{m}$ brightness temperature, $\sim 2 \%$ in the solar $24 \mu \mathrm{m}$ brightness temperature, and $2.5 \%$ of the $\alpha$ Cen A $24 \mu \mathrm{m}$ measurement). The measurement of $26.1 \pm 0.2 \mathrm{mJy}$ for $\alpha$ Cen A from Liseau et al. (2015) then suggests that the solar analog calibration is brighter than the ALMA measurement by $10.7 \%$ $\pm 7 \%$, which confirms that the $10 \%$ ALMA calibration uncertainty estimate is plausible.

If we adjust the measurement of Fomalhaut to this alternative calibration, we find a flux density of $1.98 \mathrm{mJy}$ (instead of $1.789 \mathrm{mJy}$ ), still somewhat fainter (by $\sim 11 \%$ ) than the Rayleigh-Jeans extrapolation from $24 \mu \mathrm{m}$. We conclude that radiative transfer effects may cause a drop in the brightness temperature of the star, similar to the behavior of the Sun, which has a brightness temperature $\geqslant 5800 \mathrm{~K}$ across the visible (Vernazza et al. 1976) but of only $5470 \mathrm{~K}$ at $870 \mu \mathrm{m}$. The result that Fomalhaut is slightly fainter than the Rayleigh-Jeans extrapolation value is robust, and this independent calibration of our measurement also rules out any compact free-free emission originating from the star as the possible source of excess emission detected by Spitzer and Herschel. 


\section{ANALYSIS}

\subsection{Properties of Inner 20 AU Excess in Fomalhaut}

In this subsection, we review all the available measurements that can be used to constrain the excess properties of the inner $20 \mathrm{AU}$ region in Fomalhaut. These measurements can be categorized in two classes: (I) resolved measurements where emission from the cold belt is mostly excluded, and (II) unresolved measurements at the wavelengths where the cold belt contributes much less emission. In Category I, the groundbased interferometric measurements suggest a $K$-band excess of $0.88 \% \pm 0.12 \%$ (Absil et al. 2009; hereafter the VLTI measurement) and $8-13 \mu \mathrm{m}$ excess of $0.35 \% \pm 0.10 \%$ (Mennesson et al. 2013; hereafter the KIN measurements) relative to the stellar photosphere. These interferometric observations can very effectively isolate the region that is very close to the star ( $\lesssim 2 \mathrm{AU}$ in $K$ band and $\lesssim 6 \mathrm{AU}$ at $8-13 \mu \mathrm{m}$ ) from the contribution of the stellar photosphere. In addition, the excess emission at 24 and $70 \mu \mathrm{m}$ inferred from the resolved images of Fomalhaut by Spitzer and Herschel also belongs to the first category. Since the star and any unresolved excess are both contained within the instrument's point-spread function (PSF), the excess estimate does depend on the expected photospheric values.

In determining the unresolved excess, an accurate value for the photospheric flux is essential; it can be determined at $2 \%$ levels by fitting the stellar atmospheric models to the optical to near-infrared photometry ( $\mathrm{Su}$ et al. 2005; Engelbracht et al. 2007). At $24 \mu \mathrm{m}$, an additional unresolved source of $0.6 \pm 0.2 \mathrm{Jy}$ was found in the early reduction of the MIPS data (Stapelfeldt et al. 2004). We reduced the original data with the final in-house reduction pipeline and calibration and employed the PSF subtraction method. We find that the unresolved excess at $24 \mu \mathrm{m}$ is $0.64 \pm 0.13 \mathrm{Jy}$, consistent with the old value (but with a smaller uncertainty). Acke et al. (2012) constrained the amount of the unresolved excess by fitting the resolved images with an SED model using complex dust grains (a mixture of compositions proposed by Min et al. 2011) and derived a total of $0.17 \pm 0.02$ Jy for the unresolved excess at $70 \mu \mathrm{m}$. Su et al. (2013) employed the PSF subtraction method and derived a consistent number, $0.17 \mathrm{Jy}$ with upperand lower-bound fluxes of 0.58 and $0.136 \mathrm{Jy}$, respectively, for the unresolved excess. The resolution at both Spitzer $24 \mu \mathrm{m}$ and Herschel $70 \mu \mathrm{m}$ is very similar $\left(\sim 6^{\prime \prime}\right)$, which places the excess emission mostly from the region within $\sim 20 \mathrm{AU}$ in projected stellocentric distance.

In Category II, the Spitzer IRS spectrum of the Fomalhaut system presented in Su et al. (2013) provides good measurements in the 10-30 $\mu \mathrm{m}$ region. The IRS spectrum was extracted and calibrated as a point source (resolution of $\left.\sim 6^{\prime \prime}\right)$. With a large slit size of 11 !' 1 in the IRS long-high channel, the flux longward of $\sim 30 \mu \mathrm{m}$ might be contaminated by the cold ring. Furthermore, the IRS excess emission is subject to the uncertainty of photospheric subtraction. To account for this, the uncertainty for the IRS excess emission includes $2 \%$ of the photospheric emission (added quadratically) as shown in Figure 2. To illustrate the effect of photospheric subtraction, we also present excess spectra by artificially scaling our bestdetermined photospheric model by $\pm 2 \%$. The resultant spectra (shown in Figure 2) have higher/lower flux in the 10-15 $\mu \mathrm{m}$ region, but both are within the estimated uncertainty (gray area). We augment the IRS excess determination with

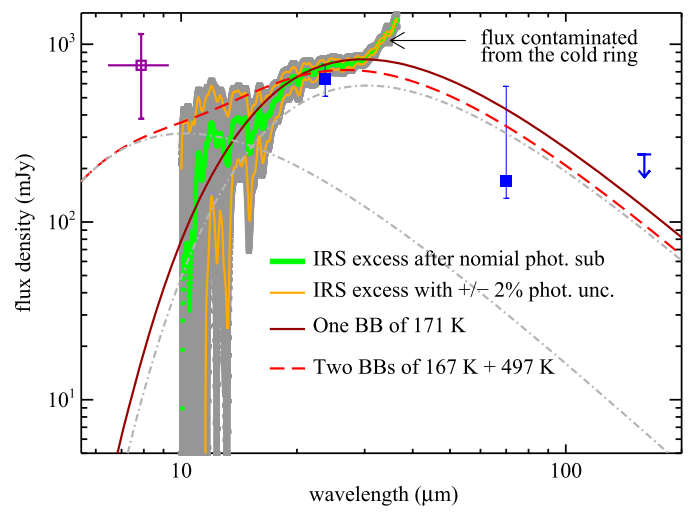

Figure 2. SED of the infrared excess for the inner $20 \mathrm{AU}$ region around Fomalhaut. The thick green line is from the IRS spectrum presented in $\mathrm{Su}$ et al. (2013) after the nominal photospheric subtraction. The gray area represents the uncertainty of the IRS excess, including $2 \%$ of the photospheric subtraction. The thin orange lines represent the excess IRS spectra if the photosphere is $\pm 2 \%$ higher/lower than the nominal value. The filled squares represent the broadband excess emission estimated from the resolved images, the open square is from the IRAC measurement (see text for details), and the $3 \sigma$ upper limit at $160 \mu \mathrm{m}$ is shown as a downward-pointing arrow. With the nominal and $2 \%$ higher photospheric subtraction, the IRS excess spectra (green and lower orange lines) are best described by a blackbody emission of $\sim 170 \mathrm{~K}$ for wavelengths shorter than $30 \mu \mathrm{m}$. If the photosphere is lower by $2 \%$, the resultant IRS excess spectrum is best described by a combination of two blackbody temperatures at 167 and $497 \mathrm{~K}$ (two gray dot-dashed lines).

additional accurate ${ }^{7}$ broadband photometry. We adopt the accurate PSF fitting photometry $( \pm 1.5 \%$ level $)$ by Marengo et al. (2009) for Fomalhaut and Sirius in the IRAC bands. The color difference between Fomalhaut and Sirius is 2.36 mag at IRAC bands 1, 2, and 3, but $2.33 \mathrm{mag}$ at IRAC band 4 , suggesting a $3 \% \pm 1.5 \%$ excess at IRAC $8 \mu \mathrm{m}$ (a very broad bandwidth of $36 \%$ ). Since the IRAC measurement was derived by PSF fitting, the excess most likely originates from the region within $8 \mathrm{AU}$ (in radius) (resolution of $\sim 2^{\prime \prime}$ at IRAC $8 \mu \mathrm{m}$ ).

One good way to characterize where the bulk of excess emission comes from is to determine the dominant dust temperature in the excess spectrum. This dominant dust temperature simply means that the majority of the dust grains have a similar dust temperature, which is not subject to a specific grain model even though this temperature is usually derived by the blackbody formula. Fitting the IRS excess emission, the inner warm emission is best described by a blackbody emission of $\sim 170 \mathrm{~K}$ (see Figure 2), and the temperature remains the same for the excess spectrum that was oversubtracted by $2 \%$ more of the photosphere. If the photosphere level is lower by $2 \%$ compared to the nominal level (i.e., undersubtracted), the resultant IRS excess emission needs at least two temperatures to fit, 167 and $497 \mathrm{~K}$. Owing to the uncertainty of the photospheric subtraction, we cannot rule out the presence of $\sim 500 \mathrm{~K}$ dust in the system, especially, the two-temperature fit is a better fit to the IRAC $8 \mu \mathrm{m}$ point. An alternative fit in this case is a single blackbody plus a weak (broad) silicate emission feature (see discussion in Section 4.3.1 and Figure 6 for some examples). Even with this tentative excess emission either as a continuum emission of

\footnotetext{
To obtain infrared excesses at the $\sim 2 \%$ level, using a color difference referenced to a clean, no infrared excess star (like Sirius) can avoid the uncertainty in photospheric subtraction as long as both the star of interest and the reference star (Sirius) have a similar spectral type and accurate photometry. Unfortunately, ALLWISE and AKARI IRC catalogs do not match the latter criterion sufficiently well.
} 
$\sim 500 \mathrm{~K}$ or as a broad feature, the $10-30 \mu \mathrm{m}$ excess can only be fitted with a cooler component at $167 \mathrm{~K}$, suggesting the robustness of the warm excess at the temperature of water ice sublimation $(\sim 150 \mathrm{~K})$, i.e., an asteroid-belt analog.

\subsection{Parameters for the Asteroid Belt Model}

We use a simple optically thin SED model to estimate the physical location of the asteroid belt. Our SED model is based on the assumption that the warm $(\sim 170 \mathrm{~K})$ excess originates from a planetesimal belt located near the water ice line. Therefore, we only use data longward of $\sim 10 \mu \mathrm{m}$ to constrain our fits and do not include the possible hot $\sim 500 \mathrm{~K}$ component (see Section 4.3 for further discussion). In this scenario, dust debris is generated through collisions of large parent bodies and cascades down to fine grains (close to the radiation blowout size) with a typical power-law slope of -3.5 to -3.7 (Wyatt et al. 2011; Gáspár et al. 2012). The radiation blowout size ${ }^{8}$ depends on the stellar fundamental parameters (mass and luminosity) and grain properties (size and density). Using astronomical silicates (Laor \& Draine 1993) with various densities ranging from 1.8 to $3.3 \mathrm{~g} \mathrm{~cm}^{-3}$ to account for porosity, the blowout size (radius) is $\sim 2.5-5 \mu \mathrm{m}$ around a 2.25 $M_{\odot}$ star with a luminosity of $17.4 L_{\odot}$. The maximum grain size is set at $1 \mathrm{~mm}$ since grains larger than this size contribute negligible emission in the infrared. We further assume that the warm dust belt is confined in a narrow ring (NR) with a fixed width of 2 AU. The exact function of the density distribution in the warm belt has little impact in the output SED for such an NR; therefore, we adopt a Gaussian profile for the ring's density distribution. Because we lack good constraints on the Rayleigh-Jeans side of the excess emission, the SED models are not well constrained even with all the assumptions mentioned before. A small range of $\sim 8-15$ AU with various combinations of minimum grain size and size distribution slope can provide satisfactory fits to the observed SED. We illustrate this degeneracy by presenting two NR models peaked at 9 and 13 AU shown in Figure 3, where both models have the same grain size distribution ( $5 \mu \mathrm{m}$ to $1 \mathrm{~mm}$ silicate grains with a size slope of -3.65 ).

Assuming that the inner belt is inclined by $66^{\circ}$ from face-on like the outer cold belt, an NR of 9 (13) AU would cover $\sim 13$ (17) beams in its circumference in our cycle 1 map. Given an rms of $26 \mu \mathrm{Jy} \mathrm{beam}^{-1}$, the corresponding $3 \sigma$ upper limit for the whole inner belt is 1 and $1.3 \mathrm{mJy}$ at $870 \mu \mathrm{m}$ for such rings. Our NR SED models give a much fainter total flux (see Figure 3), consistent with no detection. As presented in Section 3.1, there are a few $1 \sigma-2 \sigma$ blobs along the expected 13 AU ring circumference. We simulate the $870 \mu \mathrm{m}$ model image of the $13 \mathrm{AU}$ narrow belt under the same observing depth and array configuration as the cycle 1 data. Figure 4(a) shows the synthesized image without including random thermal noise. We then added the random thermal noise by adjusting the PWV parameters in simobserve until the measured noise level reaches $26 \mu \mathrm{Jy}$ beam $^{-1}$ as in our cycle 1 observation. We then applied the same procedure that generated Figure 1(b) to

\footnotetext{
8 The grain size can be parameterized by the $\beta$ value defined as the ratio of the radiation force and the gravitational force on a particle, i.e., $\beta=3 Q_{\mathrm{pr}} L_{*} / 16 \pi c G M_{*} a \rho_{g}$, where $Q_{\mathrm{pr}}$ is the radiation pressure efficiency averaged over the stellar spectrum, $L_{*}$ and $M_{*}$ are the stellar luminosity and mass, respectively, $G$ is the gravitational constant, $c$ is the speed of light, $a$ is the grain radius, and $\rho_{g}$ is the grain density. The blowout size $\left(a_{\mathrm{bl}}\right)$ is defined when $\beta=0.5$, i.e., $\frac{a_{\mathrm{bl}}}{\mu \mathrm{m}}=1.153 \frac{Q_{\mathrm{pr}}}{1} \frac{L_{*}}{L_{\odot}} \frac{M_{\odot}}{M_{*}} \frac{\rho_{g}}{\mathrm{~g} \mathrm{~cm}^{-3}}$.
}

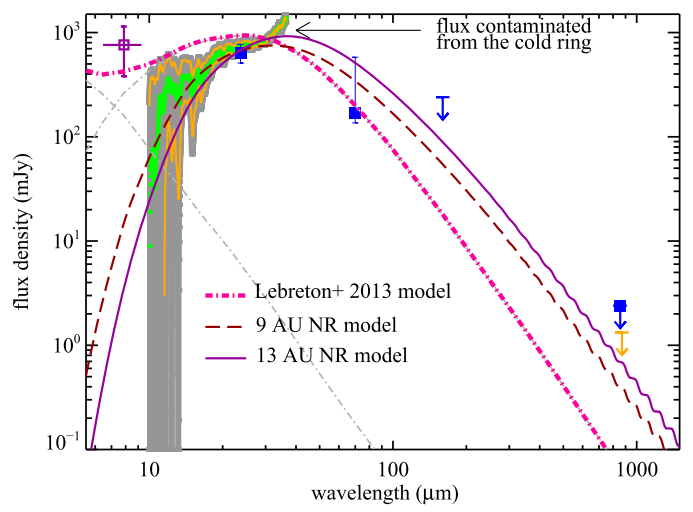

Figure 3. SED of the infrared excess for the inner $20 \mathrm{AU}$ region around Fomalhaut with various models. The data points are the same as in Figure 2, except for the ALMA Band 7 upper limits. The measurement from the cycle 0 Band 7 (Boley et al. 2012) is shown as a filled square with a downwardpointing arrow, while the one from cycle 1 is shown as an orange downwardpointing arrow. The model from Lebreton et al. (2013) is shown as the thick dot-dashed line composed of two belts (thin dot-dashed lines) at $\sim 0.1$ and $\sim 2$ AU. Our NR models are aimed to fit the excess emission near the ice line (asteroid-belt analog) and are constrained by data points longward of $10 \mu \mathrm{m}$.

generate a star-subtracted residual map of the synthesized image shown in Figure 4(b) for comparison. Note that the noise in our simulation is added randomly; therefore, it is not expected to coincide with the observation when compared to Figure 1(b). Futhermore, we did not include any background confusion as estimated by Oteo et al. (2015) using ALMA calibration data, i.e., Figure 1(b) is expected to have some contribution of faint $(\gtrsim 3)$ background galaxies. Our simulation suggests that the southeast disk ansa might be detected at $\sim 2 \sigma$ levels. Nevertheless, a deeper map is needed to confirm this putative 13 AU belt.

\subsection{Complexity of Inner Debris Distribution-a New Interpretation of Interferometric Data}

\subsubsection{A New Model for the Inner 20 AU Excess in Fomalhaut}

Our proposed asteroid belt (a narrow belt near the water ice line, presented in Section 4.2) would not produce any detectable signal in the KIN measurements because the dust in this narrow belt is too cold (i.e., too faint) at $8-13 \mu \mathrm{m}$, and it is located mostly outside the field of view of KIN. To reconcile with the KIN measurements, here we explore a different scenario including a P-R drag-in component from the asteroid belt and a hot $(\sim 1500 \mathrm{~K})$ ring produced by the magnetically trapped nanograins proposed by Rieke et al. (2015). To roughly estimate the feasibility of a P-R component from the proposed asteroid belt, we first compare the typical P-R timescale with the collisional one in our proposed ice-line belt. ${ }^{9}$ The collisional timescale is $\sim 2 \times 10^{4} \mathrm{yr}$ for a belt at $10 \mathrm{AU}$ with an optical depth of $1 \times 10^{-4}$ (see below) around a $2.25 M_{\odot}$ star. This collisional timescale is slightly shorter than the P-R timescale, $\sim 3.6 \times 10^{4} \mathrm{yr}$ for $\beta=0.5$ grains, suggesting that some amount of material can drift inward under the influence of P-R drag, forming an interior extended disk. Additional material might be deposited in this region by disintegrating comets; hence, the P-R drag component represents a rough

\footnotetext{
9 The collisional timescale is formulated as $t_{\text {coll }}=\frac{\sqrt{r^{3} G M_{*}}}{2 \tau_{\text {eff }}}$ and the P-R timescale as $t_{\mathrm{PR}}=c r^{2} / 4 G M_{*} \beta$, where $r$ is the stellocentric distance and $\tau_{\mathrm{eff}}$ is the optical depth.
} 

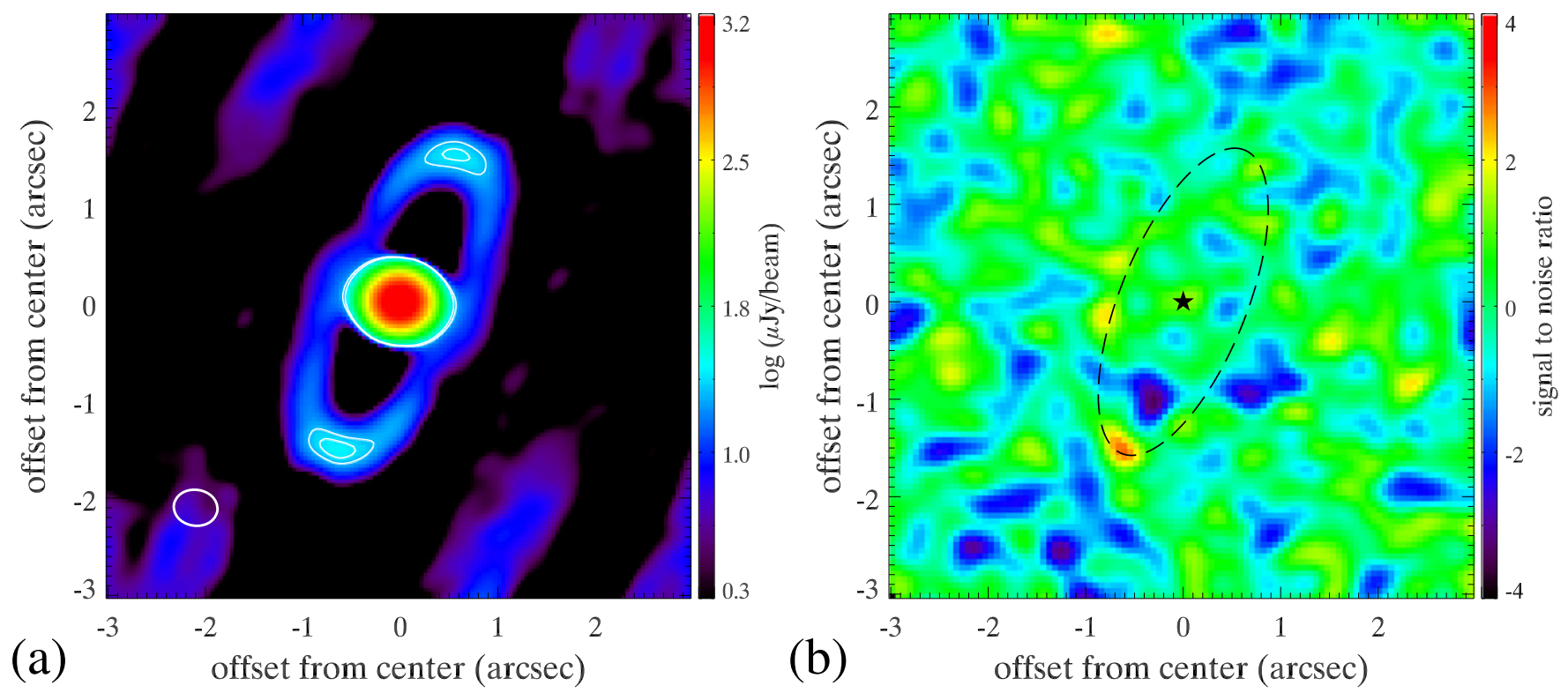

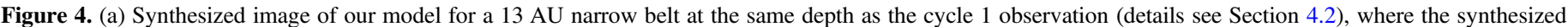

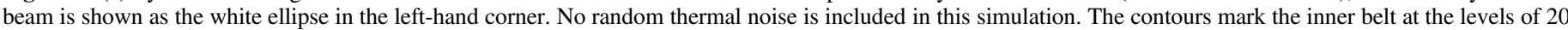

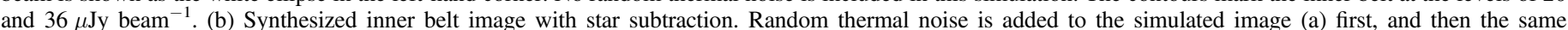

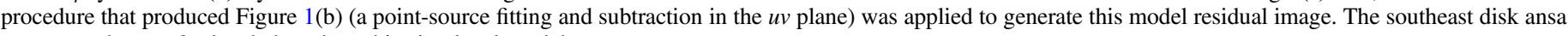
appears to be at $\sim 2 \sigma$ levels based on this simulated model.

lower limit, but one that can be analyzed without introducing uncontrolled free parameters.

We construct individual SED models for each of the components according to its spatial constraint and expected grain population in the drag-in disk. By adjusting the individual contribution of each component, we then simultaneously obtain good fits in the KIN measurements and the overall SED.

The amount of material that can be brought inward from a dust-producing planetesimal belt as a result of P-R drag has been studied analytically (Wyatt 2005) and numerically (van Lieshout et al. 2014). Basically, it depends strongly on the amount of material (i.e., the collision rate) in the planetesimal belt. Assuming a single grain size in the belt and that the collisions are destructive, the effective optical depth $\left(\tau_{\text {eff }}(r)\right.$, the vertical optical depth for a face-on disk) is parameterized as Equations (4) and (5) in Wyatt (2005). To estimate $\tau_{\text {eff }}\left(r_{0}\right)$ in Wyatt's formulae, ${ }^{10}$ we use the observed fractional luminosity $\left(f_{d}\right)$ and the relationship $\tau_{\text {eff }}\left(r_{0}\right)=2 f_{d} r_{0} / \Delta r$, given by Kennedy \& Piette (2015) (where $r_{0}$ and $\Delta r$ are the belt's location and width, respectively). The $f_{d}$ values are $\sim 1.2 \times 10^{-5}$ in our SED models presented in Section 4.2; therefore, $\tau_{\text {eff }}\left(r_{0}\right)$ is in the range of $\sim 1 \times 10^{-4}$. For a planetesimal belt at $\sim 10 \mathrm{AU}$ around a $2.25 M_{\odot}$ star with an initial optical depth of $1 \times 10^{-4}$, the value of $\eta_{0}$ (Equation (5) in Wyatt 2005) is $\sim 2$, and Equation (4), $\tau_{\text {eff }}(r)=1 \times 10^{-4}$ $\left[1+8(1-\sqrt{r / 10 \mathrm{AU}}]^{-1}\right.$, gives the maximum amount of material that can spiral inward. van Lieshout et al. (2014) performed detailed numerical simulations by including a size distribution of particles in a collision-dominated planetesimal belt and found that the amount of the material due to P-R drag is roughly a factor of 7 lower than the simple analytical calculation. As suggested by Kennedy \& Piette (2015), one can

\footnotetext{
10 The analytical model by Wyatt (2005) has very simplified assumptions: grains with one single size and emitting like blackbodies. It is then not straightforward to use the optical depth from an SED model (a size distribution of grains with imperfect absorption coefficient) in these analytical formulae.
}

simply scale the $\eta_{0}$ value by a multiplicative factor $k$ where $k=$ $1 / 7$ to match the numerical result from van Lieshout et al. (2014). In addition, van Lieshout et al. (2014) also found that the size slope in the drag-in component is expected to be steep with a wavy distribution. Following the wording suggested in Kennedy \& Piette (2015), we refer to Wyatt's analytical study as the low-collision case and van Lieshout's numerical result as the high-collision case (or collision dominated). Furthermore, Kobayashi et al. (2009) suggest that a density enhancement (a pileup effect) can occur near the dust sublimation radius of a P-R drag-in disk (i.e., the inner edge of the P-R disk). As a dust grain drifts close to the sublimation radius and starts to sublimate (i.e., reducing its size), the radiation force on the dust grain becomes stronger and temporarily halts its inward migration; therefore, a ring near the sublimation radius can form. The sublimation radius and the enhancement factors depend on the grain composition. Refractory materials like silicate and carbon grains can give an enhancement factor up to 4-6 (Kobayashi et al. 2011).

Based on the theoretical models described above, we construct the SED of the P-R component in two parts. The first part is the drag-in disk component that has a constant surface density, starting from $\sim 10$ AU (the inner boundary of the asteroid belt) to $\sim 0.23 \mathrm{AU}$ (the sublimation radius for silicate-like grains when they reach $\sim 1300 \mathrm{~K}$ ), and is composed of a population of astronomical silicates in a power-law size distribution with a slope of -5.5 , and with sizes ranging from 3 to $10 \mu \mathrm{m}$. The choices of grain size parameters follow the recipe developed by Wyatt (2005) and van Lieshout et al. (2014), where grains with $\beta=0.5$ are the most dominant sizes as the product of collisional cascades; hence, they contribute the most emission from the drag-in disk (see Figure 5 in van Lieshout et al. 2014). The second part is for the density enhancement near the silicate sublimation radius. We place an $\mathrm{NR}$ at $0.23 \mathrm{AU}$ with a width of $0.035 \mathrm{AU}$ (i.e., $\Delta r / r \sim 0.15$ ) to 


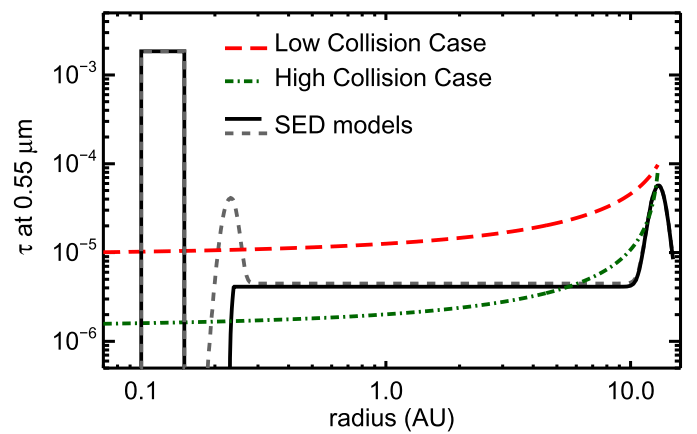

Figure 5. Optical depth in our SED models in comparison with the low collisional rate case (long-dashed line) and high collisional rate case (dotdashed line) calculations. The model with a pileup ring (upper panel of Figure 6) is shown as the dashed line, and the model without a pileup ring (bottom panel of Figure 6) is shown as the solid line. Both SED models have the same asteroid belt (at $\sim 13 \mathrm{AU}$ ) and hot ring (at $\sim 0.1 \mathrm{AU}$ ).

mimic the pileup effect. For simplicity, we adopt the same grain parameters as in the drag-in disk for this pileup ring.

We then adjust the amount of material in each of these components in the combined SED: the asteroid belt (same as before, just different normalization), the drag-in disk, and the pileup ring. To make sure these SED parameters produce a vertical optical depth distribution that is consistent with the theoretical expectation, we also compute the corresponding optical depth in the SED model as follows:

$$
\tau(r)=\frac{\sigma n d r}{2 \pi r}=\Sigma(r) \int_{a_{\min }}^{a_{\max }} Q_{\mathrm{abs}} \pi a^{2} f(a) d a,
$$

where $n$ is the dust number density and $\Sigma(r)$ is the corresponding surface density distribution (i.e., $\left.\Sigma(r)=\int n d z\right), \sigma$ is the cross section of a particle, i.e., $2 \pi a^{2} Q_{\mathrm{abs}}$ for a grain radius of $a$ and absorption coefficient $Q_{\mathrm{abs}}$, and $f(a)$ is the normalized grain size distribution. The integration limits are the grain size boundaries used in the SED model, i.e., for the drag-in disk, the limits are 3 and $10 \mu \mathrm{m}$ for astronomical silicates. We assume a constant surface density in the P-R disk; therefore, the resultant optical depth is flat for this part. Figure 5 shows the model optical depth distribution for two of the fits in comparison with the theoretical values for the low and high collisional rate cases. As shown in Figure 5, the material required in the SED fit for the P-R disk is lower than the maximum amount from the low-collision case, but higher than the collision-dominated case at distances far way from the asteroid belt.

The final component in our model is the NR that gives rise to the $K$-band excess. According to the modeling in Rieke et al. (2015), nanograins can be photoelectrically charged and magnetically trapped inside the dust sublimation radius ( $\sim 0.23 \mathrm{AU}$ for silicates or $\sim 0.1 \mathrm{AU}$ for carbon-like grains around Fomalhaut) under a nominal condition (a dipole magnetic field of $1 \mathrm{G}$ around an A-type star), and the gyroradius (i.e., the inner edge of the trapped particles) could get as close as $0.05 \mathrm{AU}$. For simplicity, we adopt a constantsurface-density ring from 0.1 to $0.15 \mathrm{AU}$, composed of amorphous carbon nanograins with sizes ranging from $0.01 \mu \mathrm{m}(10 \mathrm{~nm})$ to $0.05 \mu \mathrm{m}(50 \mathrm{~nm})$ in an $a^{-4}$ size distribution. The exact grain composition and size parameters do not have a huge impact on the SED model as long as they produce a
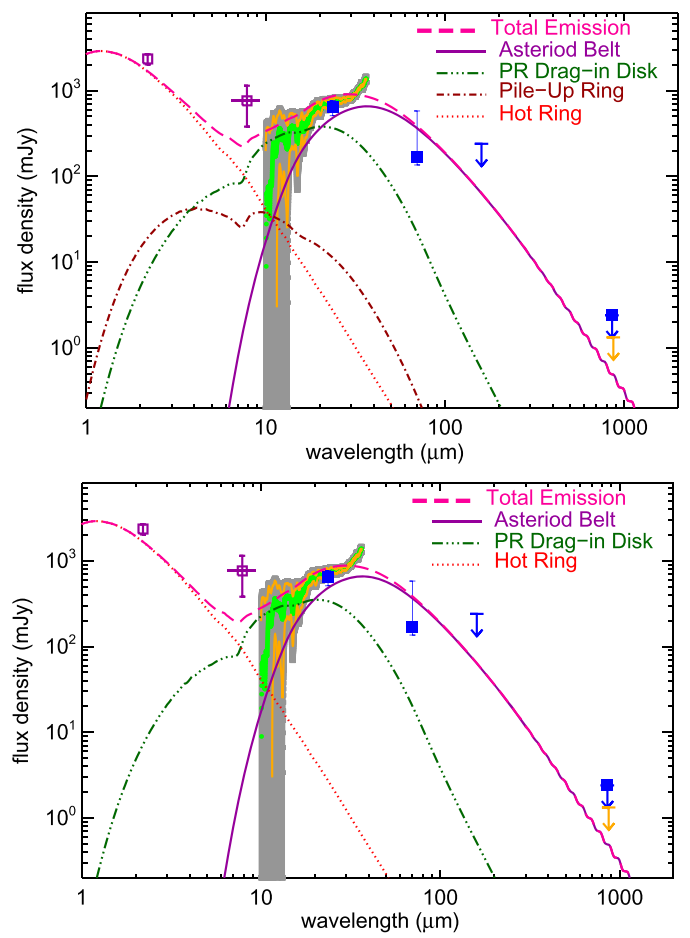

Figure 6. SED models to fit all the excess measurements in Fomalhaut's inner 20 AU region. The data points are the same as in Figure 3. The total emission (thick dashed line) is the sum of four different components: a $13 \mathrm{AU}$ narrow asteroid belt (solid line), a flat disk due to P-R drag (triple-dot-dashed line), and a hot ring composed of magnetically trapped nanograins (dotted line). The top panel shows the model that includes a narrow ring due to the pileup effect near the inner edge of the P-R disk (dot-dashed line), and the bottom panel shows the same model but without the pileup ring.

Rayleigh-Jeans-like spectrum from $\sim 2$ to $10 \mu \mathrm{m}$ that best fits the $K$-band interferometric measurement.

There are four individual components in our final SED model: the asteroid belt, the P-R disk, the pileup ring, and the magnetically trapped hot ring. As a result, many combinations of the scaling for each of the components (i.e., the total dust mass) give satisfactory fits in the overall SED, and Figure 6 shows two of them. Our final constraint comes from the spatial information in the KIN measurements. Each of the good-fit SED models produces a slightly different spatial flux distribution at the KIN wavelengths $(8-13 \mu \mathrm{m})$, i.e., different wavelength-dependent null levels (the fraction of transmitted flux detected by KIN) at different baselines (resolutions). We first simulate the high-resolution, face-on model images at $7-14 \mu \mathrm{m}$ using the best-fit SED models. Assuming that the inner dust structures have the same inclination and position angles as the cold belt $\left(i=66^{\circ}\right.$ and P.A. $\left.=156^{\circ}\right)$, the model images are then inclined and rotated accordingly. Using these model images, we then compute the expected null levels on the given dates and baselines of the KIN observations (Table 2 of Mennesson et al. 2013) and compare them with the observed levels. Figure 7 shows examples of the comparison.

By changing the combination of individual components and the associated SED parameters (like a Gaussian ring vs. a flat disk), we explore the parameter space that the KIN measurements are sensitive to. We find the following: (1) An extended dust component in the $\sim 1 \mathrm{AU}$ region (the proposed P-R disk) is needed to explain the KIN measurements, i.e., a hot nanograin ring at $\sim 0.1 \mathrm{AU}$ is not enough to produce the KIN signals, corroborating the finding in Mennesson et al. (2013). (2) The 
Table 2

Parameters in the Four SED Components

\begin{tabular}{|c|c|c|c|c|c|c|c|c|c|}
\hline Component & Dust Type & $\begin{array}{l}a_{\min } \\
(\mu \mathrm{m})\end{array}$ & $\begin{array}{l}a_{\max } \\
(\mu \mathrm{m})\end{array}$ & $\mathrm{q}$ & Density Type $^{a}$ & $\begin{array}{c}R_{\text {in }} / R_{\mathrm{p}} \\
(\mathrm{AU})\end{array}$ & $\begin{array}{l}R_{\text {out }} / R_{\mathrm{W}} \\
(\mathrm{AU})\end{array}$ & $\begin{array}{c}\text { Dust Mass } \\
\qquad\left(M_{\oplus}\right)\end{array}$ & $f_{\mathrm{d}}$ \\
\hline Asteroid belt & Silicates & 5 & 1000 & -3.65 & Gaussian Ring & 13 & 2 & $1.5 \times 10^{-5}$ & $8.8 \times 10^{-6}$ \\
\hline P-R disk & Silicates & 3 & 10 & -5.5 & Flat Disk & 0.23 & 10 & $1.4 \times 10^{-7}$ & $1.3 \times 10^{-6}$ \\
\hline Hot ring & Carbon & 0.01 & 0.05 & -4.0 & Flat Disk & 0.1 & 0.15 & $8.0 \times 10^{-11}$ & $9.7 \times 10^{-4}$ \\
\hline
\end{tabular}

Notes.

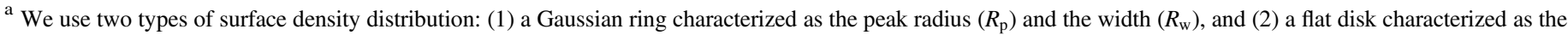
inner $\left(R_{\text {in }}\right)$ and outer $\left(R_{\text {out }}\right)$ radii.

${ }^{\mathrm{b}}$ Current data do not put a strong constraint on the existence of a pileup ring.

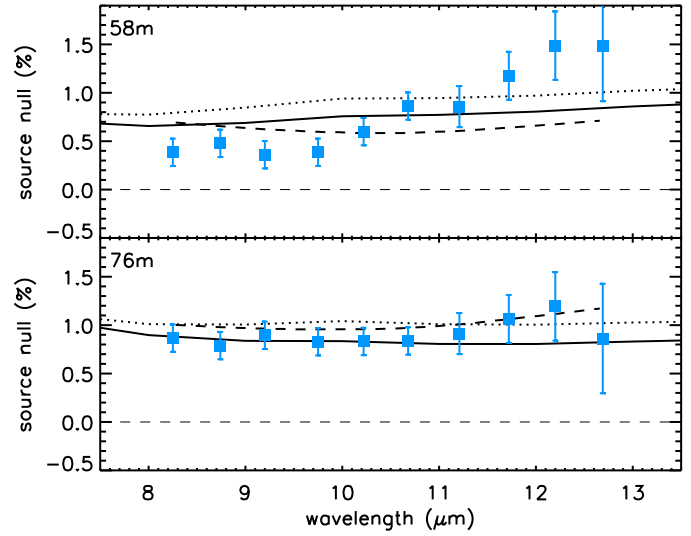

Figure 7. Null levels measured by the KIN in 2008 (squares with error bars) from Mennesson et al. (2013) in comparison with the model null levels (lines). The upper panel shows the short $(58 \mathrm{~m})$ baseline result, while the lower one is for the long $(76 \mathrm{~m})$ baseline result. Our best-fit models are shown as the solid line (without a pileup ring, i.e., the bottom panel of Figure 6) and the dotted line (with a pileup ring, i.e., the top panel of Figure 6). The null levels from the Lebreton et al. (2013) model are also shown (the thick dashed line) for comparison.

KIN measurements are not sensitive to the changes in the dust structure within $\sim 0.3 \mathrm{AU}$ (i.e., the hot and pileup rings); especially, the model with a pileup ring simply gives slightly higher null levels than those of the model without. Our SED model for the pileup ring (the upper panel of Figure 6) represents the maximum amount of emission in the model that is consistent with the overall SED. This model gives an enhancement factor of $\sim 10$ in the optical depth, which is the most extreme case found in the study of Kobayashi et al. (2011), while a typical enhancement around an A-type star for silicate grains is $\sim 3$. Since the expected emission from the maximum pileup ring is very minimal compared to the nanograin hot ring and the P-R disk (see the upper panel of Figure 6), the available measurements cannot determine whether such a pileup ring exists. (3) The wavelengthdependent behavior in the KIN short-baseline measurements (upper panel of Figure 7) is difficult to match with our simple axisymmetric model. Future observations from LBTI (Hinz et al. 2012) can help to confirm this wavelength-dependent behavior and provide complementary spatial constraints that are crucial to distinguish between degenerate KIN models (e.g., Defrère et al. 2015).

Table 2 summarizes the SED parameters in each of the components. Note that the current data do not put a strong constraint on the existence of a pileup ring near the silicate sublimation radius; we list its SED parameters just for the sake of completeness. With or without the ring due to pileup, our model appears to give a satisfactory fit to the KIN data (Figure 7), as does also the Lebreton model (details see Section 5.3).

\section{DISCUSSION}

\subsection{Does the Derived Dust Mass Make Sense?}

Is the amount of dust in the asteroid belt too much for a belt undergoing normal collisional evolution? A simple way to answer this question is to compare the observed fractional luminosity with the maximum fractional luminosity expected from collisional evolution models, $f_{\max }$ (i.e., Equation (21) from Wyatt et al. 2007). After scaling for the mass and luminosity of Fomalhaut, we find that $f_{\max }$ is $\sim 1 \times 10^{-5}$ for a belt at $10 \mathrm{AU}$ at an age of $450 \mathrm{Myr}$. The observed fractional luminosity is $\sim 0.8 \times 10^{-5}$ derived from our model, implying that the amount of dust observed in the belt does not violate the collisional evolution models. ${ }^{11}$ The $\sim 10$ AU ice-line belt can be an in situ planetesimal belt.

The optical depth distribution in our simple P-R disk model (shown in Figure 5) is between the low- and high-collision cases expected from theoretical calculations except for the region right inside of the inner belt. We also used a similar gradual curve in that part of the P-R disk, but it made no difference in the output SED and resultant KIN nulls. This is not a surprise given that this region is outside the field of view of KIN and SED models are not sensitive to changes in small spatial scale. The total derived dust mass in the P-R disk is $1.4 \times 10^{-7} M_{\oplus}$, which is $\sim 100$ times less than the derived dust mass in the asteroid belt $\left(1.5 \times 10^{-7} M_{\oplus}\right)$, suggesting that the ice-line belt can sustain the mass needed in the P-R disk. Overall, the derived dust masses in the asteroid belt and the P-R disk agree with the collisional and dynamical evolution.

For the hot ring, our derived mass is $8 \times 10^{-11} M_{\oplus}$. This value is very similar to the mass $\left(\sim 10^{-10} M_{\oplus}\right)$ derived from other studies (Absil et al. 2009; Lebreton et al. 2013; Rieke et al. 2015). In other words, the mass required to explain the VLTI $K$-band excess is on the order of $\sim 10^{-10} M_{\oplus}$. The question remains whether the P-R disk can supply enough material to form a nanograin hot ring. We can estimate the mass flow near the inner boundary of the P-R disk (without a pileup ring) as $\dot{M}_{\mathrm{PR}}(r)=\frac{L_{*}}{c^{2}} Q_{\mathrm{pr}} \tau(r)$ in the low collisional rate case (Equation (9) from van Lieshout et al. (2014)). With $L_{*}=$ 17.4 $L_{\odot}, Q_{\mathrm{pr}}=1$ for $\sim$ micron-sized grains, and $\tau \sim 4 \times 10^{-6}$ from our model, the mass flow rate is $\sim 2 \times 10^{-12} M_{\oplus} \mathrm{yr}^{-1}$ at

\footnotetext{
$\overline{11}$ The criterion is usually set when $f_{d}>100 f_{\max }$ (Wyatt et al. 2007).
} 
0.23 AU (where P-R grains sublimate). In comparison, the maximum mass flow rate from P-R drag at $0.23 \mathrm{AU}$ is $3 \times 10^{-11} M_{\oplus} \mathrm{yr}^{-1}$, using van Lieshout's Equation (11). These rates and the mass required in the hot ring suggest a typical resident time of $\sim 5-50 \mathrm{yr}$ for the nanograins. The lifetime of the trapped nanograins is estimated to be $\sim$ months to years depending on the grain sizes (Rieke et al. 2015). The estimated resident time is longer than the lifetime of nanograins if they are only supplied from the P-R disk. This implies that the P-R disk in our model can be a source to supply the nanograins; however, other mechanisms like star-grazing comets might be needed to supplement this mechanism.

\subsection{How Sensitive Are These Models to the Adopted Grain Compositions?}

The choice of the grain composition in the hot ring does not really affect the outcome of the model as long as the output SED is similar to Rayleigh-Jeans or steeper (for details see Rieke et al. 2015). We adopt amorphous carbon grains because they are a common material that has a high sublimation temperature. Other oxides like FeO can also work as well (see the discussion in Su et al. 2013; Rieke et al. 2015).

We adopt only one composition, the silicate-like grains (astronomical silicates), in both the asteroid belt and the P-R disk because they are the most common material found in interplanetary dust particles. As a result, the model SEDs show weak bumps in the 10 and $20 \mu \mathrm{m}$ silicate features because the smallest size in the model is $\sim 3 \mu \mathrm{m}$, just small enough to give rise to such features. A mixture with modest amounts of organic material would also produce a similar result with additional adjustments (like the dust sublimation radius) on the exact grain properties. The weak silicate features will be washed out when using a mixture with other featureless material. The exact numbers of the best-fit grain properties and density distribution are expected to vary, depending on the adopted grain compositions. The overall behavior in the link between the dust-producing asteroid belt and the interior, lowdensity, P-R disk remains the same.

\subsection{Comparison between Our and Lebreton's Models}

Lebreton et al. (2013) have presented the most complete and detailed model of the Fomalhaut inner debris disk to date. Since their model is different from ours, it is important to clarify what data are included in their fit and the main difference between the two models. The primary data used to constrain the Lebreton model come from the spatial constraints from the KIN 8-13 $\mu \mathrm{m}$ measurements, the VLTI $K$-band excess, and the broadband photometry at 24 and $70 \mu \mathrm{m}$. They propose two distinct dust populations in the Fomalhaut inner disk: (1) a very NR confined at $0.1-0.3 \mathrm{AU}$ from the star and composed of unbound, very small $0.01-0.5 \mu \mathrm{m}$ carbon-like grains, and (2) a disk peaked at $\sim 2 \mathrm{AU}$ with an $r^{-1}$ density distribution outward and composed of silicates and carbon grains in a very steep power-law size distribution (index of -4.8 to -4.1 ) with a minimum cutoff of $\sim 2-3 \mu \mathrm{m}$. Figure 3 also shows their model SED. This model clearly overpredicts the flux in the $15-30 \mu \mathrm{m}$ range (the IRS data were not used as a constraint), and the $24 \mu \mathrm{m}$ excess is $\sim 1 \mathrm{Jy}$ (see their Figure 5), which is higher than the unresolved excess of $0.64 \pm 0.13 \mathrm{Jy}$ estimated in Section 4.1. This is likely due to their lower photospheric estimate, $\sim 2.78 \mathrm{Jy}$ at $24 \mu \mathrm{m}$ (see their Figure 5).
Several scenarios were discussed and explored in Lebreton et al. (2013) to explain the sources of dust in the $~ 2-A U$ region, which include (1) in situ dust production, i.e., a planetesimal belt at $2 \mathrm{AU}^{12}$; (2) P-R drag-in grains from the cold $(\sim 50 \mathrm{~K})$ outer belt; and (3) planetesimal delivery scattered inward by multiple planets inside the cold belt. They ruled out the in situ dust production since the observed fractional luminosity is a few orders of magnitude higher than what a $2 \mathrm{AU}$ belt in collisional equilibrium can produce at the age of Fomalhaut (450 Myr). In comparison, the derived dust properties in our ice-line belt at the $\sim 10 \mathrm{AU}$ region are consistent with collisional evolution of an in situ planetesimal belt as discussed in Section 5.1. The P-R drag from the outer belt was also ruled out because the amount of drag-in grains is inadequate to explain the dust level at the $2 \mathrm{AU}$ region. They conclude that inward scattering of planetesimals from the outer belt by a chain of tightly packed planets is marginally adequate.

The innermost, hot ring poses more challenges as in other previous studies because its SED requires emission dominated by very small grains that are unstable against photon pressure blowout. Lebreton et al. (2013) include a detailed treatment for the dust sublimation process to produce the very small grains in the hot ring and derive a replenishing rate of $\sim 8 \times 10^{-8} M_{\oplus}$ $\mathrm{yr}^{-1}$ to sustain the hot ring. Conventional mechanisms to supply such a flow fall far short of this value; for example, Lebreton et al. find that P-R drag from the $2 \mathrm{AU}$ belt is inadequate by nearly four orders of magnitude. Even invoking an evaporating planet came up short, although the yield from such an event is very uncertain. Bonsor et al. (2014) developed the comet scattering hypothesis further and found that, with some very closely packed inner planets and an outermost planet migrating into the cold planetesimal belt, it could supply a rate slightly more than $10^{-9} M_{\oplus} \mathrm{yr}^{-1}$, still an order of magnitude too small. In addition, this hypothesis depends on an ad hoc arrangement of planets and is implausible to be operating equally well around the many other stars with similar very hot excess emission.

To mitigate these problems, Lebreton et al. (2013) proposed that the very small grains are trapped against escape by gas in the hot ring, but this solution has significant problems. Such a gas disk with normal (hydrogen-rich) abundances would be easily detected in optical emission lines (it would be analogous to the disks around Ae/Be stars; see, e.g., Mendigutía et al. 2015). Instead, Lebreton et al. (2013) suggest that the gas is the residue from the sublimation of dust, and they show that a total mass of $5 \times 10^{-3} M_{\oplus}$ would then suffice. In this case, emission in the $\mathrm{C}_{\mathrm{II}} 158 \mu \mathrm{m}$ line would be expected (Zagorovsky et al. 2010). Cataldi et al. (2015) have used Herschel/PACS to place an upper limit on the emission from this line from the entire Fomalhaut ring system, and they show that the signal from the spaxal centered on the star (see their Figure 1) is even less than from the rest of the cold ring. Assuming that the gas coincident with the very hot dust would be at a similar temperature, the upper limit is an order of magnitude lower than the required mass in the gas disk hypothesized by Lebreton et al. (2013).

In our model, the excess detected by the long-baseline KIN observation arises from the P-R drag-in grains from the $\sim 10$ AU ice-line belt. These drag-in grains then sublimate as they get close to the sublimation radius $(0.23 \mathrm{AU}$ for silicate-like grains) and become the charged nanograins trapped by the

\footnotetext{
$\overline{12}$ The typical dust temperatures in this region are $\sim 400-450 \mathrm{~K}$, which is very different from the dust temperature we refer to as the ice-line belt.
} 
weak magnetic field of the star as proposed by Rieke et al. (2015), forming the hot ring. The lifetime of these nanograins (months to years) then suggests a replenishing rate of $\sim 10^{-10} M_{\oplus} \mathrm{yr}^{-1}$. As discussed in Section 5.1, the P-R mass inflow rates are in the range of $10^{-12}-10^{-11} M_{\oplus} \mathrm{yr}^{-1}$ from the ice-line belt; an additional source of nanograins, like stargrazing comets, may be needed to supplement the P-R inflow from the ice-line belt. This star-grazing comet delivery is different from the cometary delivery scenario presented in Bonsor et al. (2014), where a very specific planetary configuration is required to sustain the high influx rate of comets. There will be a wide range of planetary configurations that both meet the scattering criteria as evaluated by Bonsor \& Wyatt (2012) and allow the scatter of a few star-grazing comets per year into the inner part of the Fomalhaut planetary system (including the tightly packed Bonsor et al. (2014) configuration).

\subsection{The Ice-line Asteroid Belt-a Natural Source for the Dust interior to the Belt}

The prominent cold belt cannot supply enough material to the inner 2 AU region as discussed by Lebreton et al. (2013). With our new parameters for the ice-line belt (dust mass of $1.5 \times 10^{-5} M_{\oplus}$ and collisional timescale of $2 \times 10^{4} \mathrm{yr}$ ), the required mass flow rate, $\sim 10^{-9} M_{\oplus} \mathrm{yr}^{-1}$, is still too high to be sustained by the outer 140 AU cold belt due to P-R drag (the maximum mass flow is $\sim 10^{-12} M_{\oplus} \mathrm{yr}^{-1}$; Equation (11) from van Lieshout et al. 2014). Unlike $\epsilon$ Eri (MacGregor et al. 2015), the $7 \mathrm{~mm}$ ATCA observation of Fomalhaut (Ricci et al. 2012) does not reveal any convincing excess emission above the photosphere. Also, ionized winds from A-type stars like Fomalhaut are extremely weak (Babel 1995). Therefore, it is unlikely that stellar-wind drag can aid the P-R drag significantly. An in situ ice-line belt, as we have demonstrated, is the best alternative to supply the interior dust detected by infrared interferometry.

The amount of interior dust that can be delivered from a planetesimal belt may even be overstated by the P-R drag estimates. In our solar system, the dust level interior to the Kuiper Belt is thought to be constant into $\sim 10 \mathrm{AU}$, where most of the particles are ejected by Saturn and Jupiter (MoroMartín \& Malhotra 2005), and only a very small amount of the P-R drag-in dust can reach Earth's vicinity (Liou \& Zook 1999; Kuchner \& Stark 2010; Vitense et al. 2010). These model predictions are consistent with the measurement by the Student Dust Counter (Szalay et al. 2013; Szalay et al. 2015) on board the New Horizons mission. In addition to the denseness of a belt (collision rate), giant planets located interior to a planetesimal belt can also reduce the amount of dust interior to the belt. To verify whether the amount of material due to P-R drag interior to a planetesimal belt is lower than the expected value (i.e., a sign of an additional depletion mechanism like the presence of a giant planet(s)), a detailed collisional cascade calculation is needed. However, such a model requires detailed information about the planetesimal belt (i.e., location and mass) that is currently lacking.

\section{CONCLUSION}

We report an ALMA cycle $1,870 \mu$ m observation centered at the star position of the Fomalhaut planetary system. We detect a point source, consistent with the bare stellar photosphere, and no extended structures that are brighter than $3 \sigma$ in the central $15 \times 15 \mathrm{AU}$ region. We evaluate all available measurements to constrain the infrared excess arising from dust in the inner $20 \mathrm{AU}$ region in Fomalhaut and conclude that a dust-producing planetesimal belt at the ice sublimation temperature (i.e., an asteroid-belt analog) is the most likely origin for the infrared excess longward at $\sim 15 \mu \mathrm{m}$. The location of the ice-line belt is estimated to be at $\sim 8-15$ AU using SED models with nominal parameters for a narrow belt with a $3 \sigma$ upper limit of total flux less than $1.3 \mathrm{mJy}$ at $870 \mu \mathrm{m}$. Assuming that the inner belt has the same orientation as the outer one (inclination and position angles of $66^{\circ}$ and $156^{\circ}$, respectively), but centered at the star position, we detect a few $1 \sigma-2 \sigma$ blobs along the expected disk circumference of a $13 \mathrm{AU}$ belt. Although our SED model suggests that such a 13 AU belt might be detected by our cycle 1 data at $1 \sigma-2 \sigma$ levels, the putative $13 \mathrm{AU}$ belt needs future confirmation.

We further propose a new coherent model to explain the interferometric hot excesses by connecting the proposed asteroid belt to the dust structures inside of it. We suggest that a small amount of material from the ice-line belt can spiral inward under the influence of P-R drag, forming an interior, extended disk composed of dust grains that have $\beta$ values closer to 0.5 . The inner boundary of the P-R disk is set by the dust sublimation radius of the dominant material like silicates ( $~ 0.23 \mathrm{AU}$ around Fomalhaut when grains reach $\sim 1300 \mathrm{~K})$. We also consider a possible pileup ring near the silicate sublimation radius as proposed by Kobayashi et al. (2009) with an enhancement factor of a few in the optical depth. We show that the required optical depth of such an interior disk (and a pileup ring) is lower than the maximum allowable amount through the P-R drag under the theoretical calculations. Finally, the sublimation of these drag-in silicate grains is likely to produce nano carbon-like or FeO-like particles that should be blown out by radiation pressure very quickly under a normal circumstance. However, a weak $(\sim 1 \mathrm{G})$ magnetic field from a fast-rotating A-type star like Fomalhaut can efficiently trap these photoelectrically charged nanograins forming a hot ring. The location of the hot ring depends on the magnetic field strength and the grain sublimation temperature. As modeled by Rieke et al. (2015), a ring at $\sim 0.1$ AU and composed of nano, very refractory grains around Fomalhaut can explain the $K$ band excess detected by VLTI. The required resident time of the nanograins sustained entirely from the P-R disk is somewhat longer than the collisional lifetime of these nanograins; therefore, additional sources like a few star-grazing comets are needed to explain the hot ring. Combining all these components (the asteroid belt, the P-R disk with and without a pileup ring, and the hot nanograin ring), we can simultaneously obtain good fits to the excess SED and satisfy the spatial constraints set by the KIN measurements.

Studying the inner zones in debris disks requires high angular resolution that can only be provided in scattered light by ground-based high-contrast imaging facilities and/or $H S T$, and by ALMA in thermal emission. However, the scattered light study of the inner debris structures around A-type stars like Fomalhaut is challenging because the inner debris is intrinsically faint due to lower mass (compared to the outer debris) and lack of efficient scatterers of small grains (typical radiation blowout size for Fomalhaut is $\sim 3 \mu \mathrm{m})$. Although future LBTI and James Webb Space Telescope mid-infrared observations can trace $\sim$ micron-sized grains in the inner zone 
in great detail, their structures are much more influenced by radiation forces (radiation pressure and P-R drag). Only ALMA can resolve the detailed structure of the asteroid belt by tracing the millimeter-sized grains that show the imprint of extrasolar terrestrial planets through dynamical interactions. Knowing the detailed properties of the asteroid belt not only will help us better understand the processes creating tenuous dust structures interior of the belt but also provides insights into extrasolar terrestrial planets that we cannot probe otherwise. Deeper observations with the full ALMA array have the potential to reveal directly the asteroid belt inferred from our SED models.

This paper made use of the following ALMA data: ADS/ JAO.ALMA 2012.0.00238.S (PI: S.P.L.). ALMA is a partnership of ESO (representing its member states), NSF (USA), and NINS (Japan), together with NRC (Canada) and NSC and ASIAA (Taiwan), in cooperation with the Republic of Chile. The Joint ALMA Observatory is operated by ESO, AUI/ NRAO, and NAOJ. K.Y.L.S. is grateful for funding from NASA's ADAP program (grant number NNX11AF73G). Support for G.H.R. is provided by NASA through contract 1255094 and 1256424 issued by JPL/Caltech to the University of Arizona. S.P.L thanks the support of the Ministry of Science and Technology (MoST) of Taiwan with Grants NSC 98-2112M-007-007-MY3, NSC 101-2119-M-007-004, and MoST 1022119-M-007-004-MY3.

\section{REFERENCES}

Absil, O., Mennesson, B., Le Bouquin, J.-B., et al. 2009, ApJ, 704, 150 Acke, B., Min, M., Dominik, C., et al. 2012, A\&A, 540, A125

Alekseeva, G. A., Arkharov, A. A., Galkin, V. D., et al. 1996, BaltA, 5, 603 Babel, J. 1995, A\&A, 301, 823

Backman, D., Marengo, M., Stapelfeldt, K., et al. 2009, ApJ, 690, 1522

Ballering, N. P., Rieke, G. H., Su, K. Y. L., \& Montiel, E. 2013, ApJ, 775, 55

Boley, A. C., Payne, M. J., Corder, S., et al. 2012, ApJL, 750, L21

Bonsor, A., Raymond, S. N., Augereau, J.-C., \& Ormel, C. W. 2014, MNRAS, 441, 2380

Bonsor, A., \& Wyatt, M. C. 2012, MNRAS, 420, 2990

Cataldi, G., Brandeker, A., Olofsson, G., et al. 2015, A\&A, 574, L1

Chen, C. H., Mittal, T., Kuchner, M., et al. 2014, ApJS, 211, 25

Chiang, E., Kite, E., Kalas, P., Graham, J. R., \& Clampin, M. 2009, ApJ, 693, 734

Defrére, D., Hinz, P. M., Skemer, A. J., et al. 2015, ApJ, 799, 42

Dermott, S. F., Jayaraman, S., Xu, Y. L., Gustafson, B. A. S., \& Liou, J. C. 1994, Natur, 369, 719

Engelbracht, C. W., Blaylock, M., Su, K. Y. L., et al. 2007, PASP, 119, 994

Engels, D., Sherwood, W. A., Wamsteker, W., \& Schultz, G. V. 1981, A\&AS, 45,5

Gáspár, A., Psaltis, D., Rieke, G. H., \& Özel, F. 2012, ApJ, 754, 74

Gáspár, A., Rieke, G. H., \& Balog, Z. 2013, ApJ, 768, 25
Greaves, J. S., Sibthorpe, B., Acke, B., et al. 2014, ApJL, 791, L11

Hinz, P., Arbo, P., Bailey, V., et al. 2012, Proc. SPIE, 8445, 84450U

Holland, W. S., Greaves, J. S., Zuckerman, B., et al. 1998, Natur, 392, 788

Johnson, H. L. 1965, CoLPL, 3, 73

Kalas, P., Graham, J. R., \& Clampin, M. 2005, Natur, 435, 1067

Kalas, P., Graham, J. R., Fitzgerald, M. P., \& Clampin, M. 2013, ApJ, 775, 56

Kennedy, G. M., \& Piette, A. 2015, MNRAS, 449, 2304

Kennedy, G. M., \& Wyatt, M. C. 2014, MNRAS, 444, 3164

Kobayashi, H., Kimura, H., Watanabe, S.-I., Yamamoto, T., \& Müller, S. 2011, EP\&S, 63, 1067

Kobayashi, H., Watanabe, S.-i., Kimura, H., \& Yamamoto, T. 2009, Icar, 201, 395

Kuchner, M. J., \& Stark, C. C. 2010, AJ, 140, 1007

Laor, A., \& Draine, B. T. 1993, ApJ, 402, 441

Lebreton, J., van Lieshout, R., Augereau, J.-C., et al. 2013, A\&A, 555, A146

Liou, J.-C., \& Zook, H. A. 1999, AJ, 118, 580

Liseau, R., Vlemmings, W., Bayo, A., et al. 2015, A\&A, 573, 4

Loukitcheva, M. A., \& Nagnibeda, V. G. 2000, in ESA Publications 463, The Solar Cycle and Terrestrial Climate, ed. A. Wilson (Noordwijk: ESA), 363

MacGregor, M. A., Wilner, D. J., Andrews, S. M., Lestrade, J.-F., \& Maddison, S. 2015, ApJ, 809, 47

Marengo, M., Stapelfeldt, K., Werner, M. W., et al. 2009, ApJ, 700, 1647

Matthews, B., Kennedy, G., Sibthorpe, B., et al. 2014, ApJ, 780, 97

McMullin, J. P., Waters, B., Schiebel, D., Young, W., \& Golap, K. 2007, in ASP Conf. Ser. 376, Astronomica Data Analysis Software and Systems XVI, ed. R. A. Shaw, F. Hill, \& D. J. Bell (Tuscon, AZ: Univ. of Arizona Press), 127

Mendigutía, I., de Wit, W. J., Oudmaijer, R. D., et al. 2015, MNRAS, 453,2126

Mennesson, B., Absil, O., Lebreton, J., et al. 2013, ApJ, 763, 119

Mermilliod, J. C. 1991, yCat

Min, M., Dullemond, C. P., Kama, M., \& Dominik, C. 2011, Icar, 212, 416

Morales, F. Y., Rieke, G. H., Werner, M. W., et al. 2011, ApJL, 730, L29

Moro-Martín, A., \& Malhotra, R. 2005, ApJ, 633, 1150

Oteo, I., Zwaan, M. A., Ivison, R. J., Smail, I., \& Biggs, A. D. 2015 , arXiv: 1508.05099

Quillen, A. C. 2006, MNRAS, 372, L14

Ricci, L., Testi, L., Maddison, S. T., \& Wilner, D. J. 2012, A\&A, 539, L6

Rieke, G. H., Blaylock, M., Decin, L., et al. 2008, AJ, 135, 2245

Rieke, G. H., Gáspár, A., \& Ballering, N. P. 2015, ApJ, 816, 50

Stapelfeldt, K. R., Holmes, E. K., Chen, C., et al. 2004, ApJS, 154, 458

Su, K. Y. L., \& Rieke, G. H. 2014, in IAU Symp., 299, Exploring the Formation and Evolution of Planetary Systems, ed. M. Booth, B. C. Matthews, \& J. R. Graham (Cambridge: Cambridge Univ. Press), 318

Su, K. Y. L., Rieke, G. H., Malhotra, R., et al. 2013, ApJ, 763, 118

Su, K. Y. L., Rieke, G. H., Misselt, K. A., et al. 2005, ApJ, 628, 487

Su, K. Y. L., Rieke, G. H., Stapelfeldt, K. R., et al. 2009, ApJ, 705, 314

Szalay, J., Piquette, M., \& Horanyi, M. 2015, LPSC, 46, 1701

Szalay, J. R., Piquette, M., \& Horányi, M. 2013, EP\&S, 65, 1145

van Lieshout, R., Dominik, C., Kama, M., \& Min, M. 2014, A\&A, 571, A51

Vernazza, J. E., Avrett, E. H., \& Loeser, R. 1976, ApJS, 30, 1

Vitense, C., Krivov, A. V., \& Löhne, T. 2010, A\&A, 520, A32

Wiegert, J., Liseau, R., Thébault, P., et al. 2014, A\&A, 563, 102

Wyatt, M. C. 2005, A\&A, 433, 1007

Wyatt, M. C., Clarke, C. J., \& Booth, M. 2011, CeMDA, 111, 1

Wyatt, M. C., Smith, R., Greaves, J. S., et al. 2007, ApJ, 658, 569

Zagorovsky, K., Brandeker, A., \& Wu, Y. 2010, ApJ, 720, 923 